\title{
A Simultaneous Forbush Decrease Associated with an Earthward Coronal Mass Ejection Observed by STEREO
}

\author{
S.Y. Oh $\cdot$ Y. Yi
}

Received: 4 April 2012 / Accepted: 18 June 2012 / Published online: 19 July 2012

(C) The Author(s) 2012. This article is published with open access at Springerlink.com

\begin{abstract}
The intensity-time profile of Forbush decrease (FD) events observed by neutron monitors (NMs) looks like that of a geomagnetic storm as defined by the Dst index. Oh, Yi, and Kim (J. Geophys. Res. 113, A01103, 2008) and Oh and Yi (J. Geophys. Res. 114, A11102, 2009) classified FD events based on the amount of overlap and simultaneity of their main phase in Universal Time (UT). Oh and Yi define an FD event as simultaneous if the main phases observed by NMs distributed evenly around the Earth overlap in UT, and nonsimultaneous if they overlap only in the local time of some stations. They suggested that the occurrence mechanisms of two types of FD events may be related to interplanetary (IP) magnetic structures such as IP shocks and magnetic clouds. In their model, the simultaneity of FD events depends on the strength and propagation direction of magnetic structures overtaking the Earth. Recently, the Solar Terrestrial Relations Observatory (STEREO) mission has been able to visualize the emergence and propagation direction of coronal mass ejections (CMEs) in three dimensions in the heliosphere; thus, it is now possible to test the suggested mechanisms. One simultaneous FD event observed on 18 February 2011 may have been caused by a CME heading directly toward the Earth, which was observed on 15 February 2011 by the STEREO mission. Therefore, the simultaneity of FD events is proven to be a useful analysis tool in understanding the geoeffectiveness of solar events such as interplanetary CMEs and IP shocks.
\end{abstract}

Keywords Interplanetary shock · Magnetic cloud · Simultaneous Forbush decrease · STEREO mission

\section{Introduction}

A sudden decrease in the galactic cosmic ray (GCR) intensity observed by ground-based detectors such as neutron monitors (NMs) and muon telescopes (MTs) is referred to as a

S.Y. Oh · Y. Yi (凶)

Department of Astronomy and Space Science, Chungnam National University, Daejeon, 305-764, Korea e-mail: euyiyu@cnu.ac.kr

S.Y. Oh

e-mail: osy1999@cnu.ac.kr 
Forbush decrease (FD) event. The intensity-time profile of FD events is similar to that of geomagnetic storms as defined by the Dst index. It evolves from the main phase of a period of decreasing intensity via a recovery phase to a steady-state level. The main phase lasts for less than half a day, while the recovery phase usually lasts several days (Forbush, 1958; Belov, 2008).

Studies of FD events such as nonperiodic GCR intensity variations enable us both to understand the processes by which interplanetary space events perturb the interplanetary magnetic field (IMF) and to explore the relevant magnetospheric and atmospheric phenomena caused by IMF disturbances (Lockwood, 1971). FD events are currently considered to result from the exclusion of GCRs due to strengthened IMF structures of the interplanetary (IP) shock sheath region and/or the magnetic clouds (MCs) following IP shocks (Venkatesan et al., 1992; Ananth and Venkatesan, 1993; Badruddin, 2002; Oh, 2008).

The global simultaneity of FD events was discussed and confirmed by Oh, Yi, and Kim (2008) and Oh and Yi (2009, 2011). They classified FD events by their simultaneity using the amount of overlap of the main phase in Universal Time (UT). Two FD events are simultaneous if they overlap in UT and nonsimultaneous if they only overlap in local time (LT). Some studies (Ahluwalia, Zubieta, and Schreier, 1968; Hofer and Flückiger, 2000) have reported LT-dependent GCR intensity variations during FD events.

Oh, Yi, and Kim (2008) and Oh and Yi (2009) focused on the onset times of the overlap of the main phases at different NMs. Their statistical studies of the simultaneity of FD events confirmed the existence of nonsimultaneous FD events. They also showed significant differences in the onset time of the main phase in LT between simultaneous and nonsimultaneous FD events, and suggested that the occurrence of FD events may be related to magnetic barriers such as IP shocks and MCs. The simultaneity of FD events in their model depends on the strength and propagation direction of magnetic barriers overtaking the Earth. Thus, here we identify the processes responsible for generating simultaneous FD events using observational data from the Solar Terrestrial Relations Observatory (STEREO) mission.

\section{Data}

STEREO is the third mission in NASA's Solar Terrestrial Probes program (Rust, 1998; Kaiser, 2005; Kaiser et al., 2008). This mission employs two nearly identical space-based observatories - one in an orbit ahead of the Earth, the other trailing behind - to provide the first ever stereoscopic measurements to study the Sun and the nature of its coronal mass ejections (CMEs). The twin STEREO spacecraft were launched on 25 October 2006 (http://stereo.gsfc.nasa.gov/).

Of the four instrument packages mounted on each of the two STEREO spacecraft, the Sun Earth Connection Coronal and Heliospheric Investigation (SECCHI; Howard et al., 2008) package can show the three-dimensional evolution of CMEs from their birth on the solar surface via the corona and the interplanetary medium to their eventual impact on Earth. This package consists of four instruments: an Extreme Ultraviolet Imager (EUVI), two white-light coronagraphs (COR1 and COR2), and a Heliospheric Imager (HI) (http://secchi.nrl.navy.mil/). We used image data from COR1 and COR2 and IMF data observed by the Advanced Composition Explorer (ACE; Smith et al., 1998). A preliminary list of CMEs observed on 15 February 2011 with COR1 (inner coronagraph) is given in Table 1. The angular separation between the two spacecraft (Behind and Ahead) was 179.1 degrees. Five CMEs were observed, and the CME observed at 02:05 UT may have been 
Figure 1 Positions of STEREO $\mathrm{B}$ and $\mathrm{A}$ relative to the Earth on 15 February 2011 at 02:00 UT. The dotted lines show the angular displacement from the Sun. HEE indicates Heliocentric Earth Ecliptic distance in AU. http:// stereo-ssc.nascom.nasa.gov/ where.shtml.
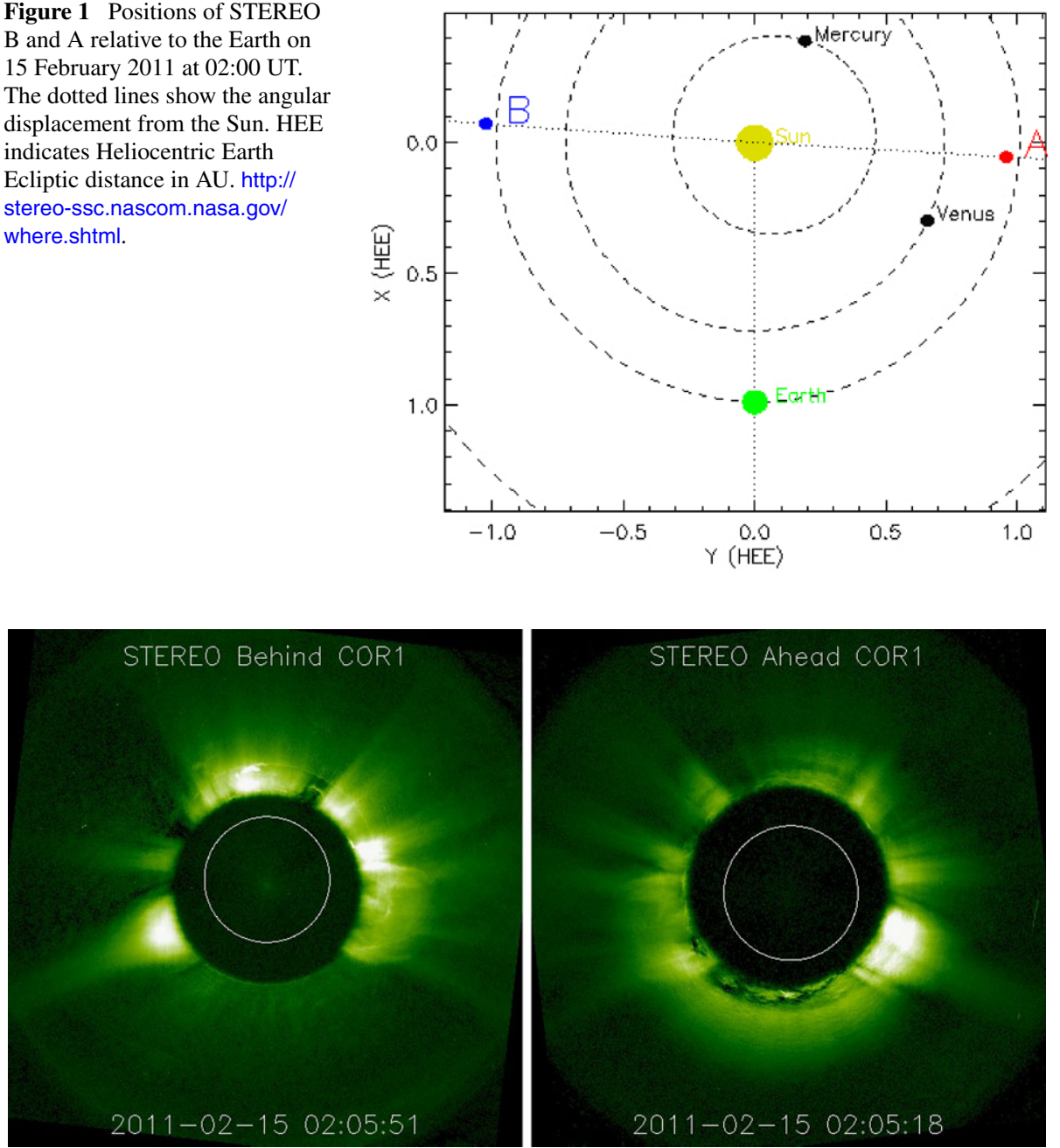

Figure 2 Images of the solar corona, taken by the SECCHI inner coronagraph (COR1) on the STEREO Behind and Ahead spacecraft on 15 February 2011 at 02:05 UT.

associated with a simultaneous FD event on 18 February 2011. It is also associated with an $\mathrm{X}$-class flare. We also compared our STEREO observations with the data observed by the Solar and Heliospheric Observatory (SOHO)/Large Angle and Spectrometric Coronagraph experiment (LASCO; Brueckner et al., 1995). To produce the time profiles of FD events, we used hourly cosmic ray data from the Oulu, Magadan, and Inuvik NM stations based on small geomagnetic cutoff rigidities at high latitude.

\section{Results}

Figure 1 shows the position of STEREO B and A on 15 February 2011 at 02:00 UT. Figure 2 shows an image of the solar corona taken by the SECCHI inner coronagraph (COR1) of the 

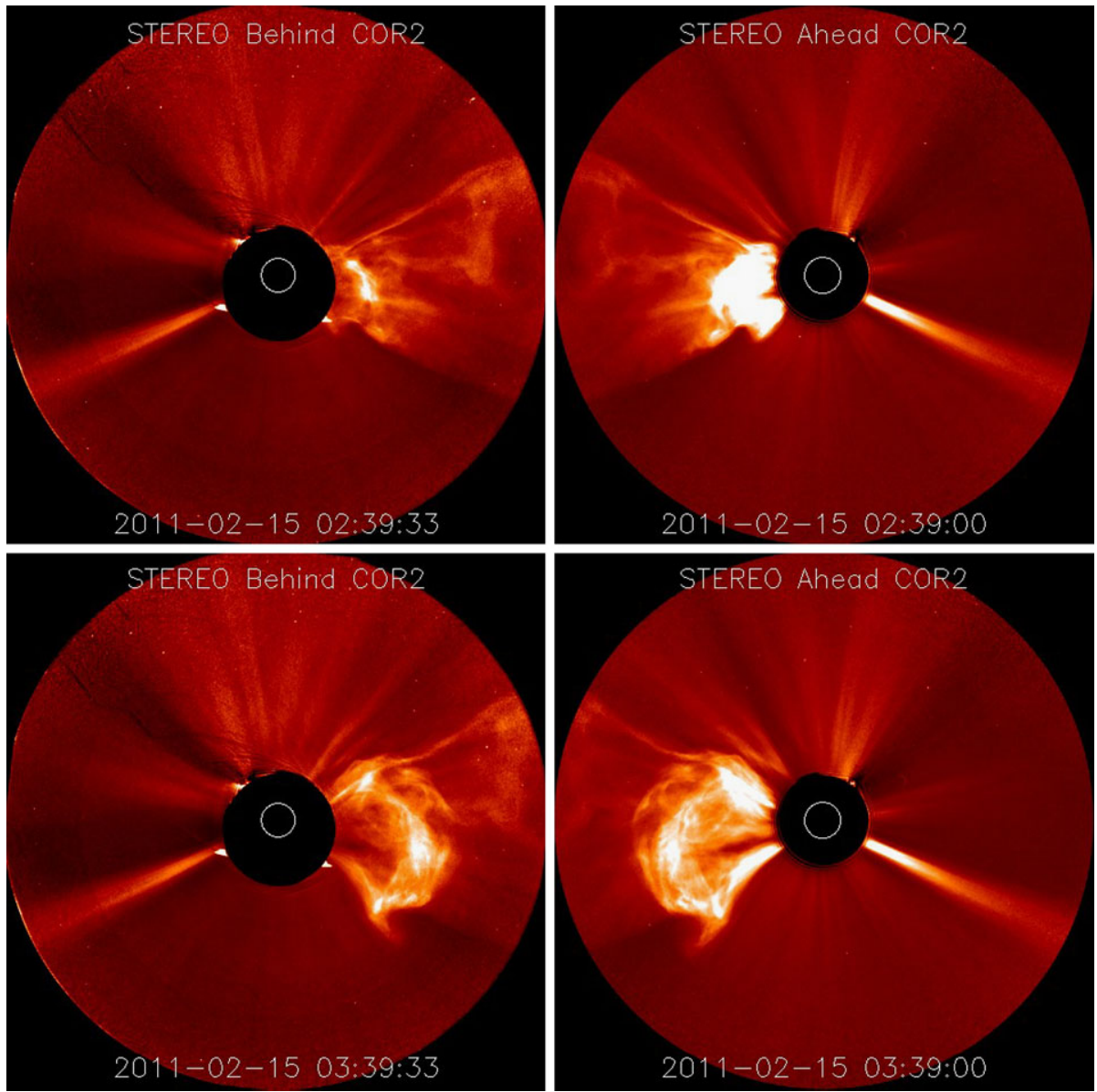

Figure 3 Images of the solar corona, taken by the SECCHI outer coronagraph (COR2) on the STEREO Behind and Ahead spacecraft on 15 February 2011 (from top left to bottom right, Behind 02:39, Ahead 02:39, Behind 03:39, Ahead 03:39 UT).

Table 1 COR1 preliminary CME list on 15 February 2011 (B-A angle separation: 179.1 degrees)

\begin{tabular}{|c|c|c|c|}
\hline UT & STEREO SECCHI COR1-B & STEREO SECCHI COR1-A & $\begin{array}{l}\text { Earthward } \\
\text { direction }\end{array}$ \\
\hline 01:05 & $\begin{array}{l}\text { W, fast eruption (flare C2.7/S18W15, } \\
\text { AR } 11158,00: 38 \text { ) }\end{array}$ & $\mathrm{E}$, fast eruption & $\mathrm{Y}$ \\
\hline 02:05 & $\begin{array}{l}\text { W, fast eruption (flare X4.8/S21W18, } \\
\text { AR } 11158,01: 56 \text { ) }\end{array}$ & $\mathrm{E}$, fast eruption & $\mathrm{Y}$ \\
\hline 05:05 & WNW, fast and narrow eruption & ENE, fast and narrow eruption & $\mathrm{N}$ \\
\hline $09: 25$ & $\mathrm{NW}$, faint and narrow eruption & $\mathrm{NE}$, faint and narrow eruption & $\mathrm{N}$ \\
\hline $15: 05$ & $\begin{array}{l}\text { NW, narrow eruption (flare C4.8/S20W } 15 \text {, } \\
\text { AR } 11158,14: 44 \text { ) }\end{array}$ & NE, narrow eruption & $\mathrm{N}$ \\
\hline
\end{tabular}




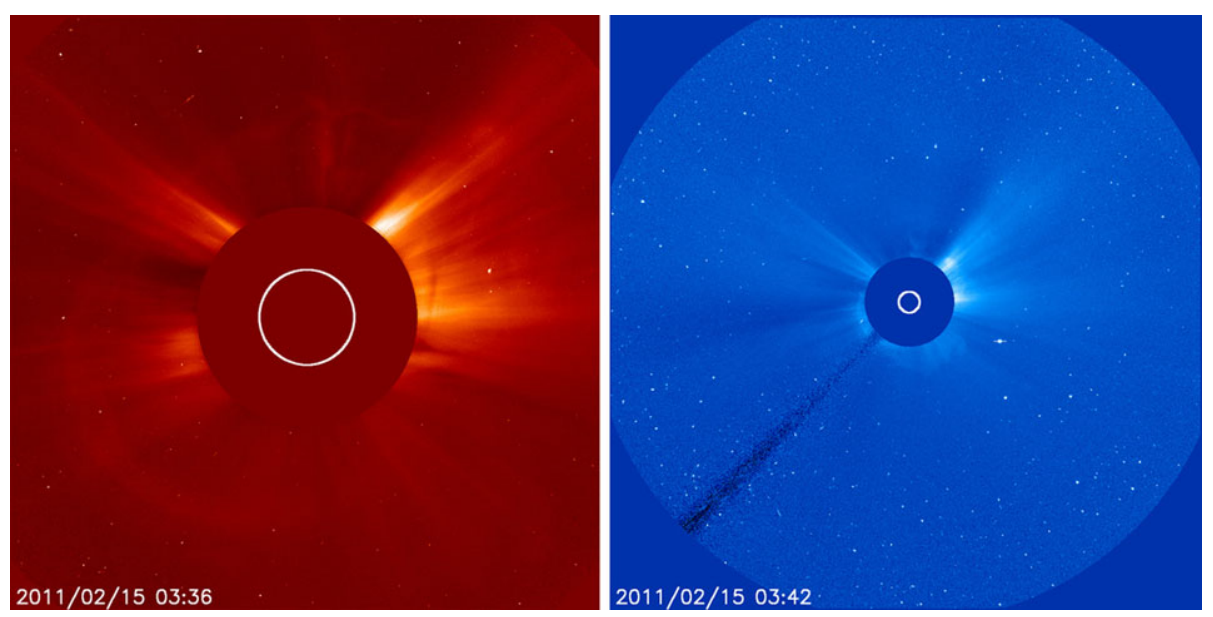

Figure 4 Images of the solar corona taken by LASCO C2 and C 3 on the SOHO spacecraft on 15 February 2011 at 03:36 UT (left) and 03:42 UT (right).

Figure 5 Time variations of GCR flux of an FD event on 18 February 2011 at the Oulu, Magadan, and Inuvik NM stations in (a) UT and (b) LT.
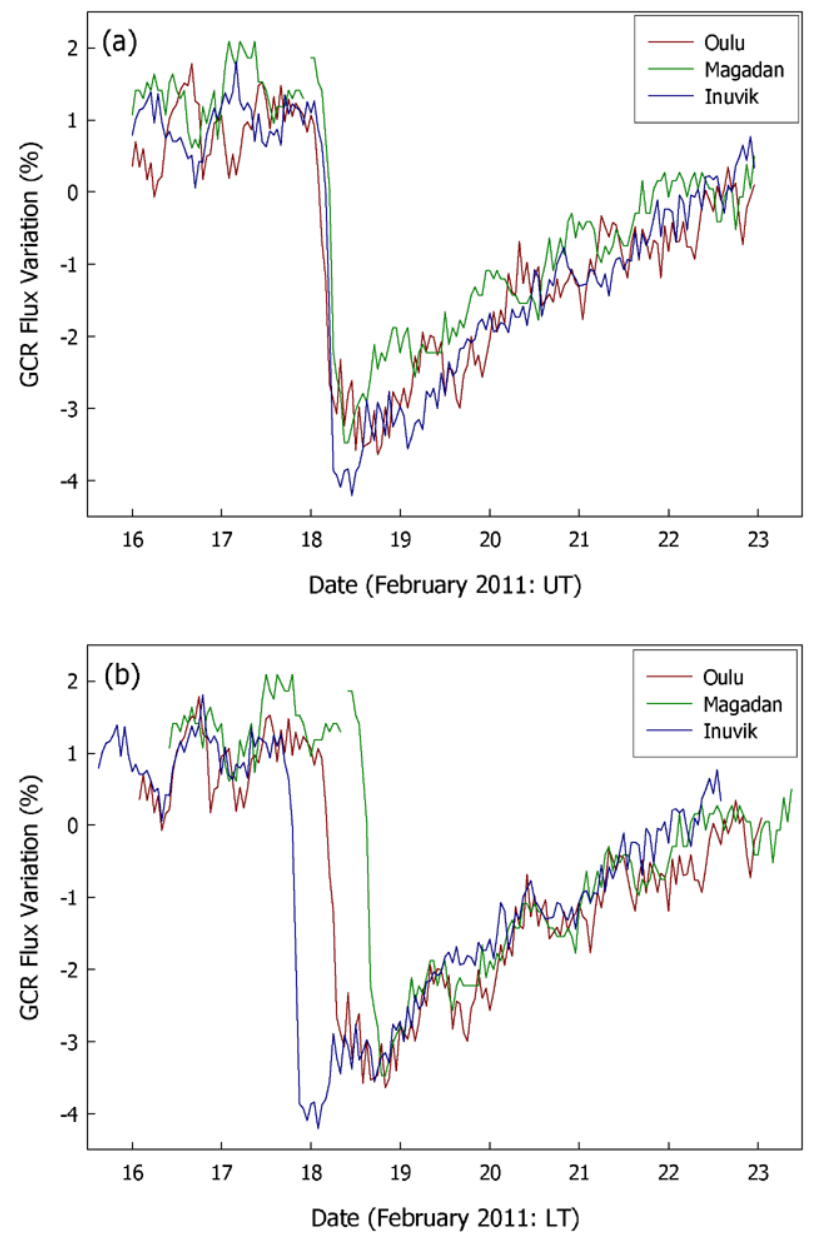
Figure 6 IMF data observed by ACE/MAG (16-23 February 2011): (a) longitude, (b) latitude, and (c) magnitude of the magnetic field. The two dashed lines (L1 and L2) indicate the IP shock and magnetic cloud, respectively.

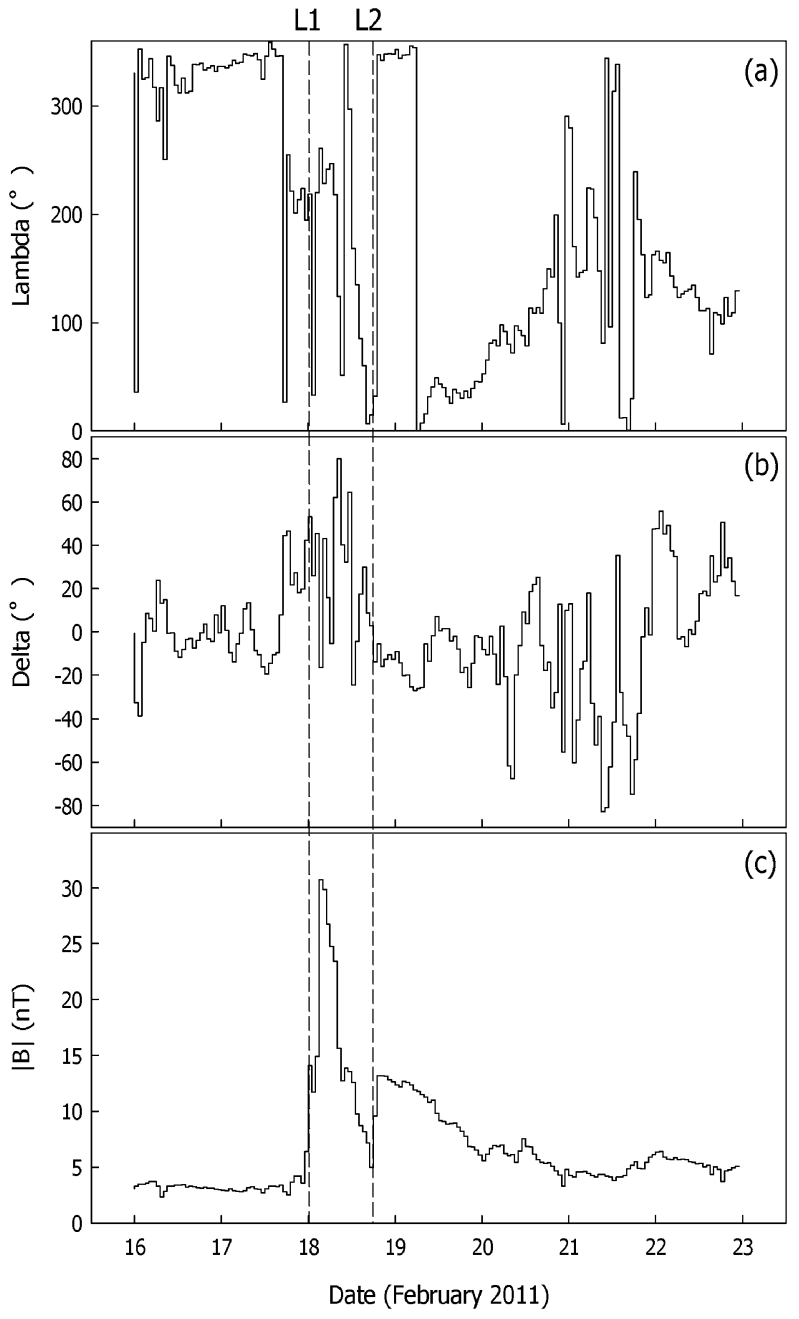

STEREO Behind and Ahead spacecraft on 15 February 2011 at 02:05 UT. The Earth is located west of Behind and east of Ahead. The CME erupted in the direction to the Earth shown in Table 1.

Figure 3 shows images of the solar corona taken by the SECCHI outer coronagraph (COR2) on 15 February 2011 at 02:09 and 03:39 UT. The symmetric CME eruption with respect to the Earth appears in COR2. Figure 4 shows images of the solar corona taken by the LASCO coronagraphs C2 and C3 on the SOHO satellite on 15 February 2011. The CME in Figure 4 is the same as that in Figure 3. The LASCO images show halo CMEs. Although they do not provide any information on their propagation with respect to the Earth, the observations by STEREO and LASCO together can confirm the propagation to the Earth.

Almost three days after the CME eruption, an FD event was observed simultaneously on 18 February 2011. Figure 5 indicates the time variations of the GCR flux of the FD event on 18 February 2011 at the Oulu, Magadan, and Inuvik NM stations in both UT and LT. The main phases at the three NM stations completely overlap in the UT profile of Figure 5a. The 
Figure 7 Schematic diagram (not to scale) of globally simultaneous FD event [redrawn Figure 7 of $\mathrm{Oh}, \mathrm{Yi}$, and Kim (2008)].

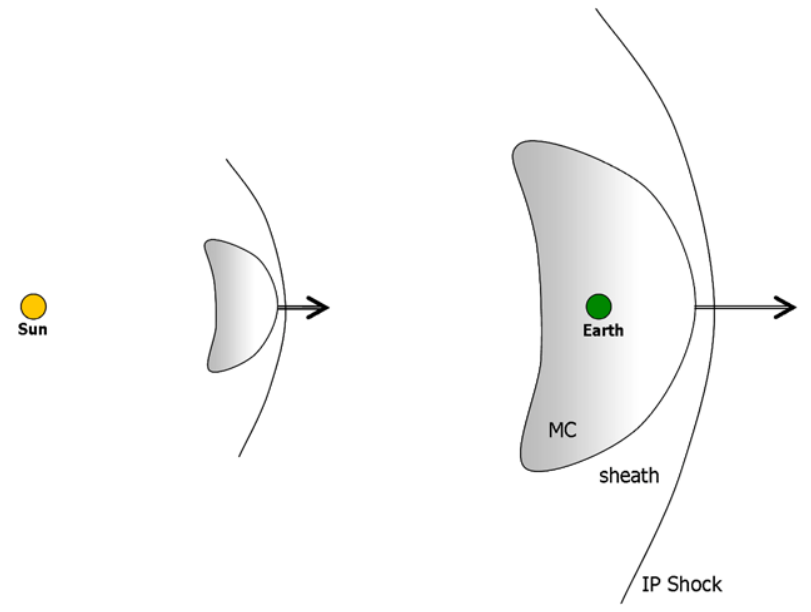

variation of the GCR intensity during the main phase is approximately $6 \%$ compared with the monthly averages.

Figure 6 shows IMF data observed by ACE/MAG during the period of 16-23 February 2011. The two dashed lines (L1 and L2) indicate the IP shock and MC, respectively. A strong magnetic field enhancement (IP shock and MC) appeared on 18 February 2011. The erupted magnetic field reached the Earth on 18 February 2011, causing a strong magnetic barrier to overtake and surround the Earth. The FD event was recorded as a simultaneous signal at the three NM stations.

\section{Summary}

We investigated the CME that occurred on 15 February 2011 as observed by the STEREO spacecraft, which might have caused the FD event on 18 February 2011 that was recorded at the Oulu, Magadan, and Inuvik NM stations. At 02:05 UT the CME erupted straight to the Earth, and it is associated with an X-class solar flare. It propagated into interplanetary space and reached the Earth on 18 February 2011; surely the central part of the interplanetary CME passed through the Earth. The FD event was detected simultaneously at all three NM stations.

Oh, Yi, and Kim (2008) suggested a model explaining a globally simultaneous strong FD event. Figure 7 is a schematic diagram explaining the formation of a globally simultaneous FD. A CME occurs at the Sun and propagates earthward. The magnetic barrier, consisting of IP shock and MC, makes a strong shield against the GCR. The GCR particles are deflected, and thus fewer GCRs can arrive at the Earth. When the central part of the magnetic barrier with a strong IMF passes the Earth, then an FD can be simultaneously recorded at different NMs. The three-dimensional visualization model of Oh, Yi, and Kim for simultaneous FD events is confirmed by this CME event, which was observed by the STEREO mission. The simultaneity of FD events has proven to be a useful analysis tool in understanding the geoeffectiveness of solar events such as interplanetary CMEs and IP shocks.

Acknowledgements This work was supported by a National Research Foundation of Korea grant funded by the Korean Government (NRF-2010-359-C00019), and also by the BAERI Nuclear R\&D program of the Ministry of Education, Science and Technology (MEST)/National Research Foundation of Korea (NRF). 
The authors are grateful to the STEREO/SECCHI team (Goddard Space Flight Center, Naval Research Laboratory), the SOHO/LASCO team (Naval Research Laboratory, Max Planck Institute for Solar System Research), the ACE/MAG team, and the ACE Science Center for providing the ACE data. The authors also thank the directors of the Oulu (Sodankyla Geophysical Observatory; http://cosmicrays.oulu.fi/readme.html), Magadan (Institute of Cosmophysical Research and Radio Wave Propagation, Russian Academy of Sciences), and Inuvik (Bartol Research Institute; http://www.bartol.udel.edu/gp/neutronm/) NMs.

Open Access This article is distributed under the terms of the Creative Commons Attribution License which permits any use, distribution, and reproduction in any medium, provided the original author(s) and the source are credited.

\section{References}

Ahluwalia, H.S., Zubieta, M., Schreier, M.: 1968, Solar Phys. 4, 453.

Ananth, A.G., Venkatesan, D.: 1993, Solar Phys. 143, 373.

Badruddin: 2002, Solar Phys. 209, 195.

Belov, A.V.: 2008, In: Gopalswamy, N., Webb, D.F. (eds.) Universal Heliophysical Processes, IAU Symp. 257, 439.

Brueckner, G.E., Howard, R.A., Koomen, M.J., Korendyke, C.M., Michels, D.J., Moses, J.D., et al.: 1995, Solar Phys. 162, 357.

Forbush, S.E.: 1958, J. Geophys. Res. 64, 651.

Hofer, M.Y., Flückiger, E.O.: 2000, J. Geophys. Res. 105, 23085.

Howard, R.A., Moses, J.D., Vourlidas, A., Newmark, J.S., Socker, D.G., Plunkett, S.P., et al.: 2008, Space Sci. Rev. 136, 67.

Kaiser, M.L.: 2005, Adv. Space Res. 36, 1483.

Kaiser, M.L., Kucera, T.A., Davila, J.M., St. Cyr, O.C., Guhathakurta, M., Christian, E.: 2008, Space Sci. Rev. 136, 5.

Lockwood, J.A.: 1971, Space Sci. Rev. 12, 658.

Oh, S.Y.: 2008, J. Astron. Space Sci. 25, 149.

Oh, S.Y., Yi, Y., Kim, Y.H.: 2008, J. Geophys. Res. 113, A01103.

Oh, S.Y., Yi, Y.: 2009, J. Geophys. Res. 114, A11102.

Oh, S.Y., Yi, Y.: 2011, J. Geophys. Res. 116, A08104.

Rust, D.M.: 1998, In: Priest, E.R., Moreno-Insertis, F., Harris, R.A. (eds.) A Crossroads for European Solar and Heliospheric Physics, ESA SP-147, 133.

Smith, C.W., L'Heureux, J., Ness, N.F., Acuna, M.H., Burlaga, L.F., Scheifele, J.: 1998, Space Sci. Rev. 86, 613.

Venkatesan, D., Badruddin, Ananth, A.G., Pillai, S.: 1992, Solar Phys. 137, 345. 